\title{
Suspensão subperiostal do terço médio da face: uma alternativa para correção do ectrópio cicatricial
}

\author{
Subperiosteal midface lift:an alternative tocorrect the cicatricial eyelid ectropion
}

Silvana Artioli Schellini ${ }^{1}$

Amilton de Almeida Sampaio Junior ${ }^{2}$
Trabalho realizado na Faculdade de Medicina de Botucatu (UNESP). Botucatu (SP).

${ }^{1}$ Professora Livre-Docente do Departamento de Oftalmologia, Otorrinolaringologia e Cirurgia de Cabeça e Pescoço da Faculdade de Medicina de Botucatu da Universidade Estadual Paulista (UNESP). Botucatu (SP). ${ }^{2}$ Ex-Residente do Departamento de Oftalmologia, Otorrinolaringologia e Cirurgia de Cabeça e Pescoço da Faculdade de Medicina de Botucatu da UNESP. Botucatu (SP).

Endereço para correspondência: Silvana Artioli Schellini. DEP. OFT/ORL/CCP - Faculdade de Medicina de Botucatu (UNESP). Botucatu (SP) - CEP 18618-970 E-mail: sartioli@fmb.unesp.br

Recebido para publicação em 04.06.2004

Versão revisada recebida em 29.09.2004

Aprovação em 22.10.2004

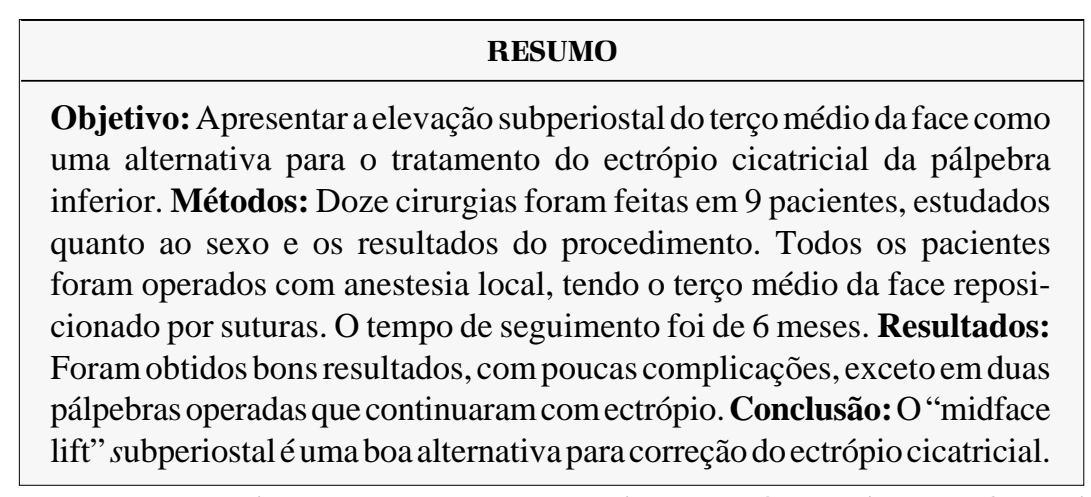

Descritores: Face/cirurgia; Doenças palpebrais/cirurgia; Pálpebras/cirurgia; Cicatriz/ cirurgia; Ectrópio/cirurgia; Músculos oculomotores/cirurgia; Cirurgia plástica

\section{INTRODUÇÃO}

O terço médio da face (midface) é uma área da face limitada superiormente por uma linha imaginária que une as duas suturas zigomático-frontais e inferiormente, no nível do implante dos dentes na maxila ${ }^{(1)}$.

A pele e a gordura subcutânea da face têm propriedades mecânicas. A espessura da gordura subcutânea varia de indivíduo para indivíduo e de uma área da face para outra. Esta gordura que está situada entre a pele e o plano muscular é dividida em lóbulos por septos de tecido conectivo(1).

Existe um outro plano de gordura entre o músculo e o periósteo, não septada, que na região malar (zygoma) é conhecida como SOOF (suborbicularis oculi fat $)^{(1-2)}$ e na região da pálpebra superior e sobrancelha é conhecida como ROOF (retro-orbicularis oculi fat) ${ }^{(1,3)}$.

Os músculos da expressão facial são interconectados por uma camada contínua fibromuscular conhecida como SMAS (superficial musculoaponeurotic system). O músculo orbicular ocular está incorporado ao SMAS ${ }^{(1,4)}$.

O envelhecimento da face pode ser analisado em três direções: inferior (sentido da gravidade), ínfero-medial (sentido do sulco nasolabial) e ântero-posterior (esvaziamento dos tecidos moles e conseqüente diminuição do volume de algumas regiões da face). A reorientação dos tecidos da face deve, portanto, ser feita nos sentidos opostos: superior, súpero-lateral e póstero-anterior $^{(4)}$.

"Midface lift" subperiostal é uma técnica que se propõe a reorientar os tecidos da região média da face, já consagrada para correção estética do envelhecimento facial, geralmente associada a outros procedimentos como encurtamento da pálpebra inferior ${ }^{(5)}$, blefaroplastia inferior ${ }^{(6)}$ ou até um "lifting" de toda a face ${ }^{(4)}$.

Usando esta técnica, existe a oportunidade de elevar o terço médio da face. 
Existe uma condição em que existe encurtamento da lamela anterior no sentido vertical e tração da pálpebra para baixo, levando ao ectrópio da pálpebra inferior. Este tipo de ectrópio é conhecido por ectrópio do tipo cicatricial e é corrigido classicamente usando retalhos e enxertos, associados ou não ao "tarsal strip"(7).

O objetivo deste trabalho é apresentar a correção do ectrópio cicatricial usando a técnica do "midface lift" subperiostal.

\section{MÉTODOS}

Doze cirurgias foram realizadas em 9 pacientes, estando os dados dos pacientes informados no quadro 1 e figuras de antes e depois da cirurgia de alguns pacientes estão apresentadas (Figuras 1, 2, 3 e 4).

A técnica cirúrgica utilizada foi a descrita (Figura 5A, B, C, D):

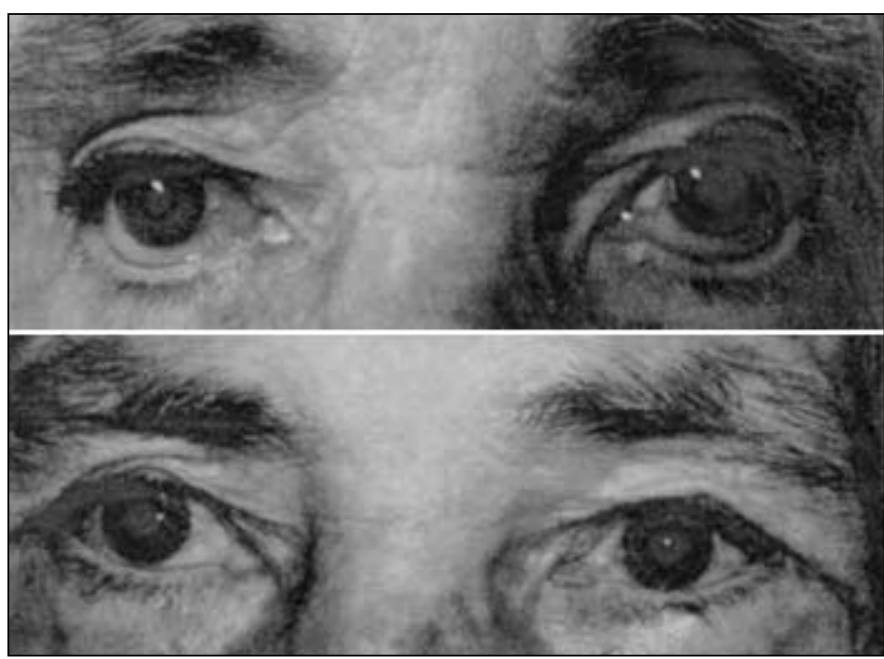

Figura 1 - Observar na figura superior, a presença do ectrópio palpebra bilateral e na inferior o reposicionamento palpebral

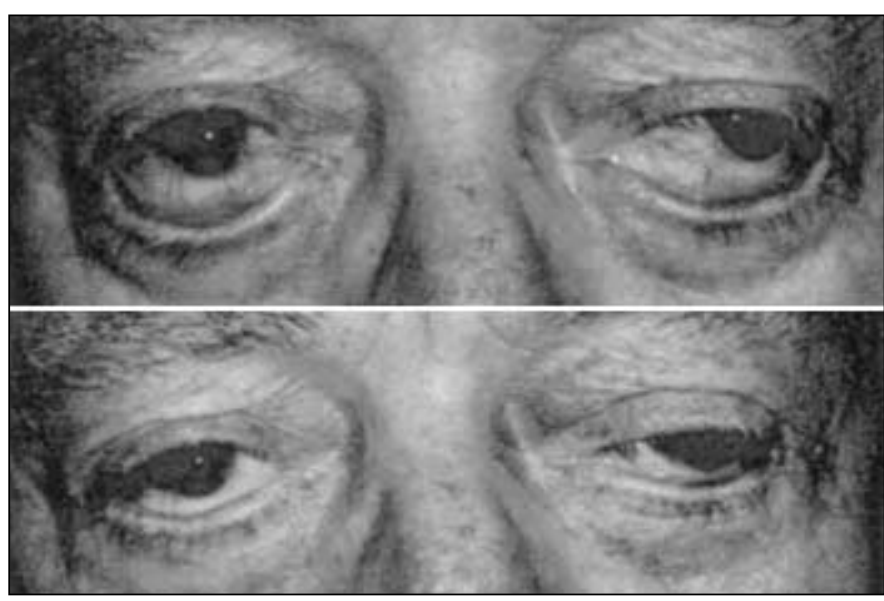

Figura 2 - Observar na figura superior, a presença do ectrópio palpebra cicatricial bilateral e na inferior o reposicionamento palpebral. 0 paciente apresenta também ptose e exotropia
- Anestesia local por infiltração de lidocaína 2\% com vasoconstrictor.

- Incisão infraciliar (1,5 mm abaixo da linha dos cílios) com bisturi lâmina 15, iniciando próximo do ponto lacrimal inferior e se estendendo até $1,5 \mathrm{~cm}$ temporal ao canto lateral palpebral.

- Dissecção com tesoura de argola sob o plano miocutâneo, sobre o septo orbitário (sem abri-lo) até expor a rima orbital inferior e lateral.

- Abertura do periósteo com bisturi lâmina 15 ao longo da rima orbital.

- Usando elevador de periósteo de Tenzel, o periósteo foi afastado dos ossos em direção inferior até no nível do implante dos dentes na maxila e nasalmente até o sulco nasolabial.

- Como todos os pacientes apresentassem também flacidez horizontal da pálpebra, associou-se o "tarsal strip", realizado com ressecção da lamela anterior, sendo confeccionada a faixa tarsal, removida a mucosa da conjuntival tarsal, suturando-se com 2 pontos separados de nylon 6-0 a extremidade da faixa tarsal até o periósteo da órbita, na região acima da posição do ligamento cantal lateral.

- Revisão da hemostasia.

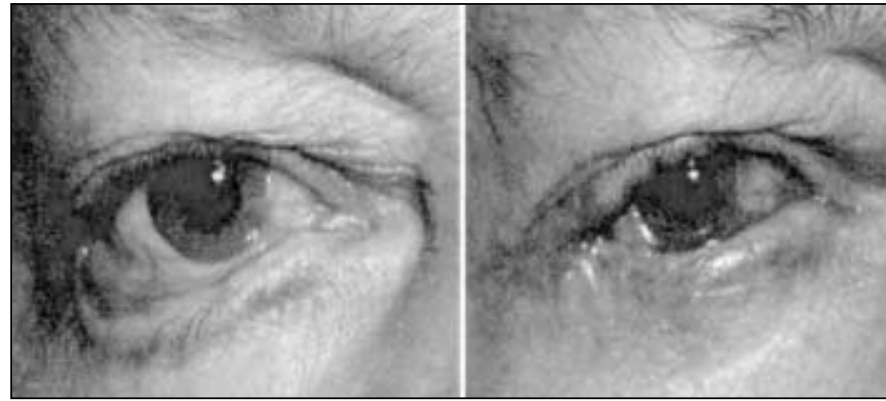

Figura 3 - Observar a esquerda, intenso ectrópio cicatricial, com exposição da conjuntiva tarsal e bulbar. Na figura da direita, o reposicionamento palpebral após a cirurgia do "midface lift", associado ao "tarsal strip"

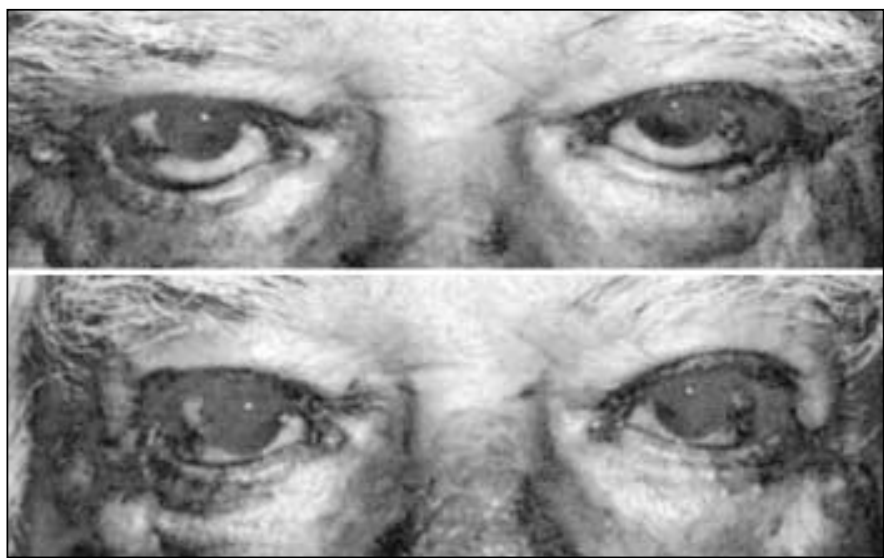

Figura 4 - Observar na figura superior, a presença do ectrópio palpebral bilateral. A figura inferior mostra o paciente no pós-operatório, com manutenção do ectrópio cicatricial e marcas visíveis da sutura de fixação feita com o nylon. $O$ paciente foi submetido também à elevação do supercílio 


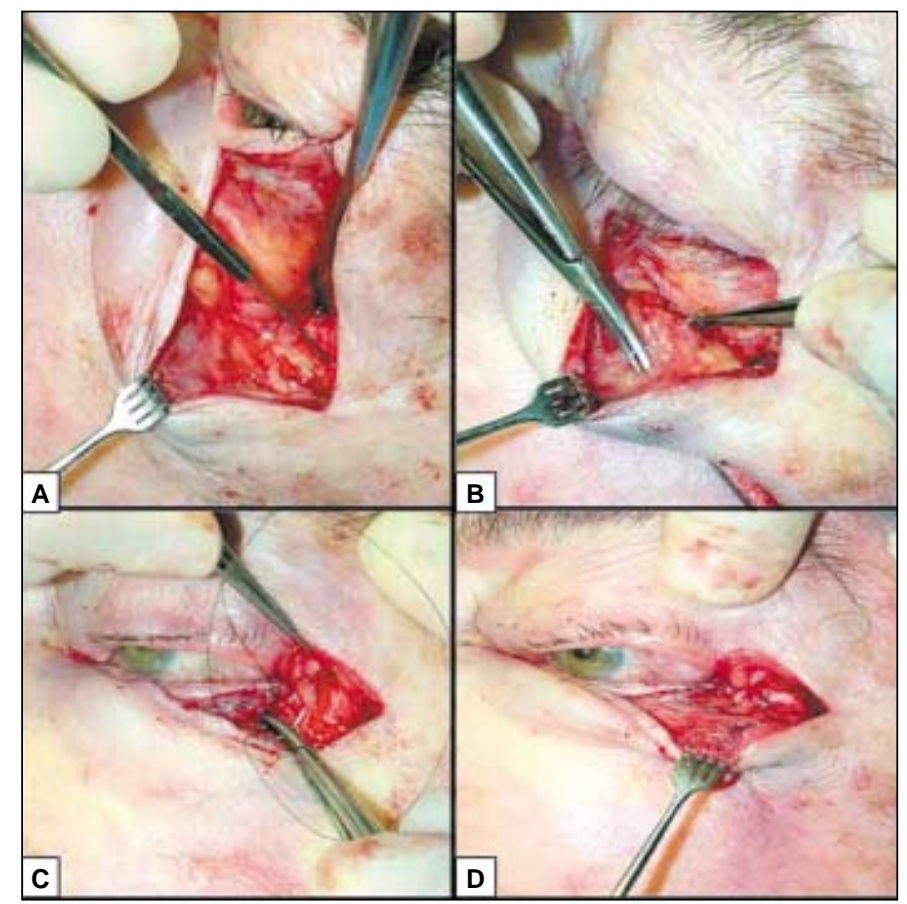

Figura 5 - Suspensão subperiostal do terço médio da face. A: Abertura do periósteo próximo a rima orbital, usando bisturi; $B$ : Passagem do fio de sutura de Nylon para fixar o SMAS; C: Local de fixação do SMAS no periósteo da porção lateral da órbita; D: Aspecto final da fixação do SMAS

\begin{tabular}{|c|c|c|c|}
\hline \multicolumn{4}{|c|}{$\begin{array}{l}\text { Quadro 1. Distribuição dos pacientes operados pela técnica do } \\
\text { "midface lifting", segundo sexo, idade e motivo da indicação da cirurgia }\end{array}$} \\
\hline Paciente & Sexo & Idade (anos) & Ectrópio \\
\hline VLM & $\mathrm{F}$ & 64 & Ectrópio- D \\
\hline VLM & $\mathrm{F}$ & 64 & Ectrópio- E \\
\hline EB & $F$ & 86 & Ectrópio- D \\
\hline EB & $\mathrm{F}$ & 86 & Ectrópio- E \\
\hline ANL & $M$ & 66 & Ectrópio- D \\
\hline AMJO & $\mathrm{F}$ & 67 & Ectrópio- E \\
\hline CVF & $\mathrm{F}$ & 63 & Ectrópio- E \\
\hline MBB & $\mathrm{F}$ & 51 & Ectrópio- D \\
\hline MBB & $\mathrm{F}$ & 51 & Ectrópio- E \\
\hline$J R$ & $M$ & 87 & Ectrópio- D \\
\hline JAS & $M$ & 70 & Ectrópio- D \\
\hline MMAP & $\mathrm{F}$ & 80 & Ectrópio- D \\
\hline \multicolumn{4}{|c|}{$F=$ feminino; $M=$ masculino; $D=$ direito; $E=$ esquerdo } \\
\hline
\end{tabular}

- Todo o terço médio da face é descolado no plano subperiostal, cuidando-se para não lesionar o nervo infra-orbital, chegando-se até o sulco nasogeniano e a implantação dentária do canino.

- O descolamento é feito também até a região da fossa temporal.

- Fixação do SMAS e do SOOF é feita com direção temporalsuperior, com vetor perpendicular ao sulco nasogeniano e feita em dois pontos separados, com fio monofilamento (nylon) 5-0, no periósteo da parede lateral da rima orbital, na região próxima ao tendão lateral e próximo a eminência malar.
- Sutura contínua da pele com fio monofilamento (nylon) 6.0.

- Curativo com pomada oftálmica (acetato de retinol, aminoácidos, metionina e cloranfenicol).

Os pacientes foram avaliados periodicamente por período de 6 meses.

\section{RESULTADOS}

A técnica cirúrgica proposta foi levada a efeito em todos os pacientes que compuseram a amostra, sem que houvesse intercorrências importantes durante sua execução.

Observou-se que, além de se obter o posicionamento da pálpebra inferior, foi possível diminuir a profundidade do sulco nasogeniano, por mobilização superior dos planos do SOOF e do SMAS.

As intercorrências intra-operatórias observadas foram: dor e sangramento (ocorrendo em um paciente hipertenso não controlado).

No pós-operatório observou-se hematoma e equimose palpebrais e da face, de intensidade não superior ao que ocorre em cirurgias do mesmo porte.

Com 6 meses de pós-operatório observou-se estabilidade e que a cirurgia proposta foi eficiente em reduzir o ectrópio palpebral em 10 das $12(83,33 \%)$ pálpebras operadas. Dois pacientes continuaram com ectrópio, tendo sido repetido o mesmo procedimento, com refixação dos tecidos em situação mais superior que a fixação inicial.

\section{DISCUSSÃO}

"Midface lift" subperiostal é uma cirurgia que segue os princípios de reorientação facial nos sentidos superior, súperolateral e póstero-anterior ${ }^{(4)}$.

Esta técnica tem sido utilizada de rotina pelos cirurgiões plásticos para a reconstrução estética do terço médio da face. Porém, não se aplica de maneira rotineira para correção do ectrópio cicatricial.

Em pacientes que apresentam ectrópio involutivo, com flacidez horizontal da pálpebra inferior, a cirurgia geralmente realizada é o "tarsal strip"(7). Se o paciente apresenta o ectrópio do tipo cicatricial ou um componente cicatricial associado ao ectrópio involutivo, comumente associa-se ao "tarsal strip" a utilização de um enxerto ou retalho de pele ${ }^{(8)}$, na tentativa de devolver à pálpebra inferior, o folheto anterior que está “faltando". Porém, este enxerto não oferece sustentação vertical, continuando atuantes as forças que tracionam a pálpebra para baixo. Além disso, com a realização dos enxertos há necessidade de duplo procedimento, lesando-se a área doadora. Acrescente-se que os enxertos são cirurgias demoradas, com vários tipos de dificuldades técnicas, com utilização de pele doadora que nem sempre tem a mesma coloração, espessura e textura da área receptora.

Utilizando o "midface lift" subperiostal, associado ou não 
ao "tarsal strip", não se faz necessário utilizar enxerto de pele e, além disso, tem-se sustentação vertical, no sentido superior e súpero-lateral dos tecidos que antes tracionavam a pálpebra para baixo.

Outra utilização para esta técnica, além da causa estética, seria para reconstrução palpebral após a remoção de tumores. Este procedimento é muitas vezes complexo, podendo ser necessário utilizar enxerto de pele para reconstruir a lamela anterior, juntamente com a mobilização do músculo orbicu$\operatorname{lar}^{(9)}$. Utilizando o "midface lift" subperiostal e, dependendo da quantidade de tecido necessário para a reconstrução que se consegue mobilizar, pode-se dispensar a utilização dos enxertos. Além disso, dispensa a mobilização de tecido muscular, já que o "lift" eleva a pele juntamente com o tecido muscular e o SOOF, diminuindo a chance de lesão das terminações nervosas, uma vez que estas estão justamente na região entre a musculatura e o $\mathrm{SOOF}^{(10-11)}$. Existe, ainda, a possibilidade de corrigir o sulco nasogeniano profundo que se instala pelo deslocamento inferior dos tecidos da face que ocorre com o passar da idade.

Os procedimentos foram realizados com anestesia local, com infiltração loco-regional, sem a administração de nenhum sedativo. Este tipo de anestesia pode ser associada à sedação, ou se utilizar a anestesia geral $^{(12)}$.

O sangramento com esta técnica não é excessivo, embora tenha ocorrido em um paciente que teve formação de hematoma e resolução do quadro por punção, conforme sugerido por outros $^{(11)}$. Ressalta-se, assim, a importância de hemostasia eficiente durante o ato cirúrgico, para que se evite os hematomas que podem prejudicar o resultado do procedimento.

Durante o seguimento dos pacientes, observou-se recidiva do ectrópio palpebral em duas das pálpebras operadas. Quando existe ectrópio muito intenso (decorrente de doenças da pele, queimaduras e trauma extenso), flacidez horizontal associada ou intensa hipoplasia do malar, retração e ectrópio da pálpebra inferior podem acontecer novamente ${ }^{(5)}$. Um encurtamento horizontal da pálpebra inferior na sua porção temporal vai estabilizá-la e diminuir o risco de ectrópio no pósoperatório $^{(5)}$.

Vale a pena lembrar que outras técnicas também podem resultar em novo ectrópio palpebral após a devida correção, surgindo meses ou anos após a primeira cirurgia, uma vez que a estabilidade da pálpebra pode ser quebrada por continuidade da retração cicatricial da pele palpebral, exposta aos mesmos fatores agressores que existiam no ambiente previamente à cirurgia.

O ectrópio pode ocorrer após blefaroplastia por outras causas, como a denervação do músculo pré-tarsal, contração cicatricial do septo orbitário e por falha na suspensão do "midface lift"(13). A falha da suspensão pode estar associada à falha técnica na execução do procedimento.

Assim, a utilização de fios de sutura inelásticos e a fixação dos tecidos no periósteo que também é uma estrutura inelástica, garantem maior chance de sucesso com o procedimento.

A localização das suturas é outro ponto muito importan- te ${ }^{(14)}$. É necessário atentar para dois pontos principais na fixação que se pretende: o primeiro é o vetor perpendicular ao sulco nasogeniano que vai garantir a elevação do terço médio facial, com a fixação na eminência malar. O segundo, é a colocação dos tecidos de forma adequada na porção média da região infra-orbital, com a fixação no canto lateral externo, próximo ao ligamento cantal. Estas duas suturas podem ser ajustadas, colocando-se maior ou menor tensão, avaliando-se durante a execução do procedimento.

Assim, observou-se que o "midface lift" coloca os tecidos do terço médio da face em uma posição mais normal, corrigindo o deslocamento inferior da região malar; promove distensão da pele do terço médio da face, eliminando algumas rugas e diminuindo o sulco nasogeniano. Além disso, os bolsões de gordura, assim como as depressões das rimas palpebrais, ficam menos aparentes pois há um reforço das estruturas que estão em um plano anterior ao septo orbitário. E oferece pele extra para a pálpebra inferior, sendo útil em pacientes com ectrópio cicatricial ou que necessitem de pele para reconstrução palpebral.

\section{CONCLUSÃO}

"Midface lift" subperiostal é uma cirurgia já consagrada para correção estética do envelhecimento do terço médio da face. Os autores enfatizam a sua utilização como alternativa efetiva também para correção do ectrópio palpebral do tipo cicatricial.

\section{ABSTRACT}

Purpose: To show the subperiosteal midface lift as an option to treat cicatricial eyelid ectropion. Methods: Nine patients submitted to 12 surgeries to correct cicatricial eyelid ectropion, were evaluated according sex, age and treatment results. All the patients had the surgery done under local anesthesia and the midface was repositioned by sutures. Results: According to our observations only two eyelids did not have good improvement with the procedure and few complications occurred. Conclusion: The subperiostal midface lift is effective and a good option to correct the cicatricial eyelid ectropion.

Keywords: Face/surgery; Eyelids diseases/surgery: Eyelids/ surgery; Cicatrix/surgery; Ectropion/surgery; Oculomotor muscles/surgery; Plastic surgery

\section{REFERÊNCIAS}

1. Putterman AM, editor. Cosmetic oculoplastic surgery: eyelid, forehead, and facial techniques. $3^{\text {rd }}$ ed. Philadelphia: W. B. Saunders; 1999.

2. Aiache AE, Ramirez $\mathrm{OH}$. The suborbicularis oculi fat pads: an anatomic and clinical study. Plast Reconstr Surg. 1995;95(1):37-42. Comment in: Plast Reconstr Surg. 1996;97(2):484-6.

3. Lemke BN, Stasior GO. The anatomy of eye brow ptosis. Arch Ophthalmol. 1982;100(6):981-6 
4. Ramirez OM. Full face rejuvenation in three dimensions: a [quot] face-lifting [quot] for the new millennium. Aesthetic Plast Surg. 2001;25(3):152-64.

5. Putterman AM. Cheek and midface lift combined with full-thickness temporal lower eyelid resection. In: Putterman AM, editor. Cosmetic oculoplastic surgery: eyelid, forehead, and facial techniques. $3^{\text {rd }}$ ed. Philadelphia: W.B. Saunders; 1999. p.235-47.

6. Trepsat F. [Lifting and blepharoplasty]. Rev Prat. 1995;45(5):557-64. French.

7. Anderson RL, Gordy DD. The tarsal strip procedure. Arch Ophthalmol. 1979; 97(11):2192-6

8. Keillor RB, Peart DA, Molteno AC. Pedicle flaps for cicatricial ectropion. Aust N Z J Ophthalmol. 1988;16(4):303-7.

9. Naugle TC, Levine MR, Carroll GS. Free graft enhancement using orbicularis muscle mobilization. Ophthalmology. 1995;102(3):493-500.
10. Finger ER. A 5-year study of the transmalar subperiostal midface lift with minimal skin and superficial musculoaponeurotic system dissection: a durable, natural-appearing lift with less surgery and recovery time. Plast Reconstr Surg. 2001;107(5):1273-83; discussion 1284. Comment in: Plast Reconstr Surg. 2001;108(6): 1824

11. De Cordier BC, de la Torre JI, AlHakeen MS, Rosenberg LZ, Costa-Ferreira A, Gardner PM, et al. Rejuvenation of the midface by elevating the malar fat pad: review of technique cases, and complications. Plast Reconstr Surg. 2002; 110(6):1526-36; discussion 1537-40.

12. Hwang K, Lee DK, Lee EJ, Chung IH, Lee SI. Innervation of the lower eyelid in relation to blepharoplasty and midface lift: clinical observation and cadaveric study. Ann Plast Surg. 2001;47(1):1-5; discussion 5-7.

13. Owsley JQ, Zweifler M. Midface lift of the malar fat pad: technical advances. Plast Reconstr Surg. 2002;110(2):674-85; discussion 686-7.

\section{SIMPÓSIO DE OFTALMOLOGIA PROF. IVO CORRÊA MEYER}

\section{Centro Científico do Hospital Mãe de Deus PORTO ALEGRE - RS}

\section{LOCAL: Anfiteatro Irmã Maria Jacomina Veronese}

Av. José de A lencar, 286 - Sub-solo

IN FO RMAÇÕES: Tel.: (51) 3346-3636 e 3395-3088

E-mail: correameyer1@brturbo.com.br 